\title{
The clinical link of preschoolers' picky eating behavior with their growth, development, nutritional status, and physical activity in Iraq/Kurdistan region
}

\author{
Khajik Sirob Yaqob Qazaryan ${ }^{1 *}$ and Saad Kazim Karim ${ }^{2}$ \\ ${ }^{1}$ Department of pediatrics, Associate membership of the RCPCH, FRCPCH, membership of Oxford university hospitals, member of American academy of nutrition and \\ dietetics, Zakho General Hospital, Kurdistan, Iraq \\ ${ }^{2}$ Consultant Neurologist, Department of Neurology, Azadi Teaching Hospital, Duhok, Iraq
}

\begin{abstract}
Background: This study aimed to evaluate the prevalence of picky eating among preschoolers and to estimate the clinical association between eating behavior and growth, physical activity, development, and health status.

Methods: In this study, a structured questionnaire was used to perform a cross-sectional descriptive study of 800 parents of preschoolers aged 2-4 years in Kurdistan/ Iraq. Data collected included: demographics, food preferences, eating behavior, body weight, BMI, height, development, physical activity, and records of medical illness. Data from children defined as picky or non-picky eaters responses were analyzed and compared using standard statistical tests according to parental' questionnaire.

Results: The mean age of the children was 2.85 years; among 800 participants, 620 (77\%) were picky eaters. Compared with non-picky eaters 180 (23\%), $z$-score of weight-for-age, height-for-age, and body mass index (BMI)-for-age in picky eaters was $0.91,0.73$, and 0.44 SD lower, respectively. There were significant variations of rates in the weight-for-age, height-for- age, and BMI-for-age percentiles $<15$, between picky and non-picky eaters $(P=0.04,0.023$, and 0.005 , respectively). Certain findings were higher in picky as compared to non picky preschoolers including negative social communication such as afraid of unfamiliar places $65 \%$ vs $13.3 \%$, afraid of being lonely $14.6 \%$ vs $12.1 \%$, poor physical activity $36.8 \%$ vs $17.7 \%$, learning disability $16.2 \%$ vs $7 \%$, attention deficit $11.8 \%$ vs $4.3 \%$, speech delay $4.6 \%$ vs $3.3 \%$, respectively).
\end{abstract}

Conclusion: The prevalence of picky eaters in preschool children was high, resulting in significant detrimental impacts on growth, nutritional status, development, physical activity, and health status.

\section{Background}

Picky or selective eating often refers to those children with strong food preferences, consuming an insufficient variety of foods, restricting the intake of certain food groups, consuming a limited amount of food, or being reluctant to try new foods. Picky-eating behaviors are common in infancy and childhood [1]; however, there is no specific medical definition for the term "picky or selective eater" [2-4].

The prevalence of selective eating among children varies in different countries. In one study in San Francisco, almost twenty to sixty percent of young children were reported by their parents not to be eating optimally [5]. Another study of 120 children aged 2-11 years identified thirty nine percent as picky eaters [6], and picky eating prevalence as high as fifty percent was reported in children aged 1924 months in a study carried out in North America [7]. Picky eating behavior was reported by parents in half $(54 \%)$ of the aged 3-7 years children in China [8]. A recent study reported that thirty six percent of young Chinese preschoolers aged 2-3 years old had selective-eating behaviors [9].

Moreover, contradictory outcomes of prevalence of childhood picky eaters were reported studies, probably due to variations in definitions, methods of assessment, and diverse age ranges of children studied $[1,6,10,11]$. Preschoolers often use their body language or non-linguistic verbalizations to express their meal favors, while older children independently make their food preferences at school, therefore the parent understand the refusal of food as being stronger as the child grows. However, picky eating behaviour is quite frequent in school children with the prevalence ranging from thirteen percentforty seven percent in developed countries $[1,6,10]$.

Picky eaters usually have a limited dietary variety and consume few fruits, vegetables and meat rich in micronutrients [12]. In addition, their intake of fats, fibre, protein and sweets is lower than that of nonpicky eaters [2]. It is still unclear whether the impact of picky eating on height and weight depends on the types of food rejected by the picky eaters.

${ }^{\star}$ Correspondence to: Khajik Sirob Yaqob Qazaryan, Department of pediatrics, Associate membership of the RCPCH, FRCPCH, membership of Oxford university hospitals, member of American academy of nutrition and dietetics, Zakho General Hospital, Kurdistan, Iraq, Tel: 0627331707; Fax: (062) 7330113; E-mail: khajikyaqob@yahoo.com

Key words: picky eating, preschoolers, growth and development, physical activity, health status

Received: November 22, 2019; Accepted: November 29, 2019; Published: December 02, 2019 
Picky eaters might have normal development besides to those with medical or developmental disorders [5], and selective eating in early childhood might extend and proceed to eating disorders in adolescence and early adulthood [13]. Furthermore, behavioral feeding disorders may be associated with delayed development $[8,14,15]$, and some children who are picky eaters might be underweight $[3,8,15,16]$.

Globally, picky eating habits of preschool children are not well studied. Currently, there has been limited research on picky eating in preschoolers with limited informative data.

In addition, fussy eaters have certain distinguished characteristics reluctance to try new foods, a dislike of certain varieties of foods, and a very good opinions about food preparation $[1,2,12]$, which result in eating small quantities and a limited types of food, potentially impacting a child's growth $[17,18]$. Consequently this can result in long-term eating disorders in adolescence and early adulthood. Hence, childhood picky eating have reported conflicting results, possibly due to inconsistencies in definitions and methods of assessment, as well as different age ranges of children studied [19]. Prevalence, studies $[1,6,10,12]$.

Some picky eating behavior in very young children, from parents' subjective perceptions, may be due to Neophobia, which is different from pickiness in older children. Neophobia is the fear of anything new, especially a persistent and abnormal fear. In its milder form, it can manifest as the unwillingness to try new things or break from routine. In the context of children the term is generally used to indicate a tendency to reject unknown or novel foods.

High nutrient requirement is needed for school-aged children as they undergo rapid growing; therefore, their eating habits are essential for optimal development. However, picky eating behavior is relatively common during childhood while at school, with the prevalence ranging from thirteen percent to forty seven percent in developed countries $[1,6,10]$.

Moreover, selective eating in early childhood has been shown to continue into mid-adolescence, which is associated with eating disorders, lasting fussy eating, and limited dietary variety in adolescence and adulthood [20-22]. However, the clinical impact of picky eating on the growth of children is still controversy. One longitudinal study of 1498 children aged 2.5, 3.5, and 4.5 years in Québec found that picky eaters were twice as likely to be underweight at 4.5 years old than children who were never picky eaters [23], whereas, Contradictory findings from another longitudinal study with 120 children in the San Francisco Bay area followed from 2 to 11 years of age suggested no significant effects of picky eating behavior on growth [6]. These opposite results might be due to certain reasons: $1^{\text {st }}$, the differences in perceptions and assessments of picky eating, $2^{\text {nd }}$, failure to adjust various confounding factors including age, gender, birth weight of the child, and socio- demographics.

Cognition and intellectual status of school children is very relevant, and is often concerning for parents. Certain studies indicated that nutrition during early childhood had long-lasting impacts on the intelligence of children $[24,25]$. As the brain grows and develops faster than the rest of the body, nutrient deficiency, especially protein, iodine, iron, zinc, folic acid, and vitamin B 12, at a critical stage of development may result in everlasting changes in brain structure and cognition status [25].

In comparison to non-picky eaters, selective eaters usually have a restricted dietary variety and limited consumption of few fruits, vegetables, and meat rich in micronutrients [12]. Moreover, their intake of fats, fibre, protein and sweets is lower than that of non-picky eaters [2]. It is unclear whether the impact of picky eating on height and weight depends on the variety of food rejected by the picky eaters. However, in one study a lower intake of vitamin $\mathrm{E}$ and $\mathrm{C}$, and fibre was found in picky nine-year-old girls [2].

\section{Subjects and methods}

This is a cross-sectional descriptive study used a structured questionnaire to obtain information from parents in Iraq/Kurdistan region who were parents to children aged 2-4 years. Participants were randomly selected from Zakho General Hospital-Department of Pediatrics, Nutrition Rehabilitation Center and private clinic for general pediatrics, child's nutrition and growth in Zakho-Duhok city in Iraq to meet pre-specified allocations for race, age, and gender, representative of the national population. A total of 800 participants (400 from each place) who met the eligibility criteria were enrolled and included in this study. Consent forms were obtained from each preschoolers' parents or caregivers.

\section{Interview process}

The interviews were scheduled, managed and enrolled by the authors themselves. Children aged 2-4 years old who had history of chronic illnesses, that might negatively impact their food behavior, were excluded for instance: prematurity, low birth weight $(<2,500 \mathrm{~g})$, dental diseases, organic diseases, mental disorders such as cerebral palsy, genetic diseases, psychiatric illness, anorexia, gastro-esophageal reflux disease, esophagitis, food allergies, and lactose intolerance. As well as those preschoolers with acute illness such as flu or diarrhea were all excluded.

Data for this study were collected between September, 2018 and July 2019. All participants gave their written and informed consent signed by their legal guardians. Using a multi-stage stratified cluster sampling method, 800 children aged 2-4 years were recruited. Data collection were obtained in this study through face-to-face interviews with the children's parents. The participants were all eligible to share in this study.

In addition, participants with low or limited economic means to support their children's diets, had inadequate concept for children's nutritional support, development, and physical activities, or were unable to provide adequate nutrition for other reasons, were also excluded in this study.

\section{Socio-demographics and anthropometric measurements}

The authors, after confirming eligibility, contacted families and arranged a meeting with parents for a face-to-face interview. The socio-demographic information was collected from the parents with a structured questionnaire survey (mothers: $99 \%$ of parents), and was administered by trained interviewers. Demographic data included child's date of birth, gender, ethnicity, and birth weight. As well as Data including any medical history of child's food allergy history and parents' body weight and height were also considered from the interview.

All participants were interviewed to gather socio-demographic data, as well as weight and height for age. During interview process we considered food preferences, eating behaviors, general development, physical activity, health questionnaire, and health status by questionnaires. The interviews took approximately $20-30 \mathrm{~min}$ to 
complete. Socio-demographic data were collected using a parental form, which included questions about educational level and number of children in the family.

The food, general health, and medical questionnaires mostly comprised closed-ended categorical questions, with listed options for respondents to select. The main questions included children's food favors, dietary habits, parent/child interactions during mealtimes, language ability, developmental behaviors, level of physical activities, and records of medical illness in the last year. As the survey was quantitative, no detailed discussions were conducted. The author carried out the interviews between the $1^{\text {st }}$ of April and the 31of June, 2019. A written informed consent were obtained from All parents of children who were participants in this study.

\section{Growth and nutritional assessment of preschoolers}

Children's weight and height were measured in Zakho General Hospital-Nutrition Rehabilitation Center-Department of pediatrics on an individual and solicited basis, before the interview associated with the food frequency questionnaire. Children were weighed without shoes and wore light clothing. These measurements were used to derive body mass index (BMI) [weight $(\mathrm{kg}) /$ height $\left(\mathrm{m}^{2}\right)$ ]. To evaluate the impact of picky eating on growth and nutritional status, the percentile and $z$-score of weight-for-age, height-for-age, and BMI-for-age were considered.

Weight-for-age, weight-for-height, and height-for-age were expressed as gender and age- specific percentiles, and the growth standards for height, weight, and BMI based on a general WHO population were used to obtain $z$-scores for each measurement according to age and sex [26].

The used parameters to assess growth status were height-for-age and weight-for-age $z$-scores, and those used to evaluate nutritional status were weight-for-age and BMI-for-age $z$-scores. Weight-for-age, height-for-age, and BMI-for-age percentiles $<15$ were considered to indicate that the child was underweight, of short stature, or suffering from malnutrition, correspondingly.

\section{Assessment of picky eating}

The questionnaire for food preferences and eating behaviors addressed two general areas (food preferences and eating behaviors).

\section{Food preferences}

In an organized questionnaire, all parents were questioned about their child's food preferences. Questions about preferences for food and food variety included a modified version of a questionnaire based on the United Kingdom Department of Health Survey of the Diets of British School Children [27] and dietary assessment among schoolaged children [28]. The modification of the questionnaire was based on Iraqi dietary culture and food habits. The questionnaire included two major items: (1) child's foods (meals) and their preferences in seven food categories: (i) grains (rice, bread, cereals, potato, noodles, etc.), (ii) protein foods (meats, fish, seafood, beans, etc.) (iii) vegetables, (iv) fruits, (v) dairy foods (milk, cheese, yogurt, etc.),

(vi) fats and oils (vegetable oil, butter, cream, salad, etc.), and (vii) snacks and sweets (candy, cookie, cake, etc.) in past 15 days, (2) preferences in common foods (list of 50 foods for regular meals).

The responses were "tried and not tried" in each food and responding to preferences of the tried foods. Items were scored on a five-point scale as "like very much," "like moderately," "neither like nor dislike," "dislike moderately," and "dislike very much."

\section{Feeding and eating behaviors}

A separate section asked parents about their feeding behaviors (six items: four appropriate behaviors, two inappropriate behaviors) and their child's eating behaviors (six items: two healthy eating behaviors, four picky eating behaviors). The eating behavior questionnaires were inspired from the Children's Eating Behavior Questionnaire developed by Wardle, et al. [29], the classification of feeding disorders of infancy and early childhood by Chatoor and Ammaniti [13] and a study about the trends of eating behaviors in preschool children [30]. The four questions for picky eating behaviors included (i) eats limited foods (usually eat fixed foods or have strong like with regard to food, such as cooked foods, milk, or sweets), (ii) unwillingness to eat regular meals, (iii) unwillingness to try new foods, and (iv) refusal of one or multiple food groups in six major food groups (grains, protein foods, vegetables, fruits, dairy foods, and fats and oil). Items were scored on a five-point scale as "never," "rarely," "sometimes," "often," or "always." Mean scores were calculated for each subscale (range 1-5) with higher scores indicating higher values of each trait.

A positive response of "always" to at least one item of the picky eating behaviors on questionnaire of eating behaviors was defined as picky eating.

\section{Assessment of development}

The questionnaire for assessment of development was modified based on the Denver Developmental Screening Test II. The test assesses children from 2 to 4 years old who are apparently asymptomatic. The DDST-II consists of 125 items grouped into four areas: personal- social, fine motor, gross motor and language. Additionally, each recording sheet includes a behavioral test in which several items are recorded, such as the child's interests or capacity to pay attention, among other items. Upon completion of the test, three scores or classifications are possible: normal, suspect and not testable [31].

The DDST-II has been adapted and emphasized in Singapore, Iran and Sri Lanka [32].

The Sensitivity and specificity values in Spanish version of the test were 89 and $92 \%$, respectively [33]; and more reliable in Hallioglu, et al. study found sensitivity values of $100 \%$ and specificity of 95 percent [34]. DDST II for early identification of the infants who will develop major deficit as a sequel of hypoxic-ischemic encephalopathy [11].

\section{Assessment of physical activity}

A modified questionnaire for assessment of physical activity was used based on a study of objective measurement of physical activity and sedentary behavior [35]. Statements relating to physical activity requested respondents to rate their degree of agreement on a five-point scale (unacceptable, improvement expected, acceptable, exceeding expected, outstanding). Mean scores were calculated for each subscale (range 1-5) with higher scores indicating higher values of each trait. The questionnaire assessing physical activity consisted of four items: normal-pace walking; sport activities; stair-climbing; and running. The answers of "unacceptable" or "improvement expected" of the item assessed were considered as having low level physical activity. Those with two or more low-level physical activities were defined as having a poor general physical activity level. 
Qazaryan KSY (2019) The clinical link of preschoolers' picky eating behavior with their growth, development, nutritional status, and physical activity in Iraq/ Kurdistan region

\section{Results and findings}

\section{Data and characteristics}

In this cross sectional study, eight hundred preschoolers aged 2-4 years were screened, of whom 740 met eligibility criteria were enrolled. Sixty participants were excluded, 30 preschoolers had chronic illnesses affecting eating habits and growth status, 15 caregivers had limited economic means to support their children's diets, and caregivers did not have enough concept for children's nutrition support, development, and physical activities or were unable to provide adequate nutrition for other reasons.

Table 1 illustrates the demographic variations between picky and non-picky eaters. Based on the food and dietary questionnaire survey, 620 preschoolers (77\%) were found to have picky- eating behavior. The mean age of these children was $2.97 \pm 0.59$ years. Certain factors such as gender, age, primary caregiver, education levels of caregiver, or family size between the participants have no statistical variations in this study.

In this study, \{always\} responders among picky preschoolers cohort were commonly as follows: Eating sweets or snacks instead of meals(52.6\%), Refusing food, particularly fruits and vegetables (37.8\%), Reluctant to eat regular meals $(27.6 \%)$, Do not like to try new food (Neophobia, 23.3\%), Ingestion of specific kinds of food (16\%), Excessive drinking of milk (14.2\%). Seventy eight cases among preschoolers disliked meat (9.7\%), vegetables (180 cases, $22.8 \%$ ), fruit (62 cases, 7.7\%), and certain types of fruit or vegetables $(52 \%, 220$ cases).

\section{Anthropometric data}

As shown in Table 2 below, in this study in Iraq/Kurdistan region, for preschoolers, the standard weight, height, and BMI (Body Mass Index) were $12.4 \mathrm{~kg}, 91.66 \mathrm{~cm}$, and 14.93, correspondingly. In comparison to non-picky eaters, such results for picky eaters were interestingly low. As well as picky eaters had significantly lower average weight-for-age, height-for-age, and BMI-for-age percentiles; Besides, less than fifty was the mean weight- and height-for-age percentiles in picky eaters, while it was greater than fifty (median of population) in non-picky eaters. Picky preschoolers also stated that they had, compared to non-picky eaters, higher rates of weight- for-age, height-for-age, and BMI-forage percentiles less than fifteen. Considerably greater percentages of children with a weight-for-age percentile less than 15 , a height-for-age percentile $<15$, and a BMI-for-age percentile $<15$ were picky eaters respectively, Table 2). The same scenario was with the $\mathrm{z}$-scores, as the average $z$-scores of weight-for-age, height-for-age, and BMI-for-age in picky eaters were all obviously less than those $z$-scores of non-picky eaters (Table 2).

\section{Development}

Table 3 displays differences in the development between picky eaters and non-picky eaters. Attention deficit and low learning ability were the detected low quality developments in learning ability. Slow verbal development and poor language development were the detected low quality developments in verbal ability. Afraid of unfamiliar places and afraid of being alone were the detected low quality developments in interpersonal relationships. Table 3 below demonstrates the prevalence rate of three categorized low quality developments in picky and non-picky group children. Of the three categories of low-quality development, a significantly higher prevalence of children who had negative interpersonal relationships was found in the picky group. A higher rate of "afraid of unfamiliar places" was reported to be picky
Table 1. Demographic variations between picky and non-picky eaters

\begin{tabular}{|l|c|c|}
\hline No of participants (800) & $\begin{array}{c}\text { Picky preschoolers (620) } \\
77 \%\end{array}$ & $\begin{array}{c}\text { Non Picky Preschoolers } \\
(180) 23 \%\end{array}$ \\
\hline Age & & $75(9.3 \%)$ \\
\hline $2-3$ y (475) & $220(27.5 \%)$ & $105(13 \%)$ \\
\hline $3-4$ y (325) & & $10(1.2 \%)$ \\
\hline Gender & $360(45 \%)$ & $170(21.2 \%)$ \\
\hline Boys (370) & $260(32.5 \%)$ & $58(7.2 \%)$ \\
\hline Girls (430) & $682(85.2 \%)$ & $6(0.75 \%)$ \\
\hline Caregivers & $54(6.7 \%)$ & \\
\hline Mother (740) & & $90(11.2 \%)$ \\
\hline Father (60) & $510(63.7 \%)$ & $35(4.3 \%)$ \\
\hline Education level & $75(9.3 \%)$ & $25(3.1 \%)$ \\
\hline Housewives $(600)$ & $65(8.1 \%)$ & \\
\hline College (110) & & $60(5 \%)$ \\
\hline Masters/PhD(90) & $170(21.2 \%)$ & $60(7.5 \%)$ \\
\hline Family size & $520(65 \%)$ & \\
\hline 3 children (210) & & \\
\hline$>3$ children (580) & & \\
\hline
\end{tabular}

Table 2. Growth and nutrition health status among pre-schoolers

\begin{tabular}{|l|c|c|}
\hline Growth and nutritional status & Picky eaters (n=620) & $\begin{array}{c}\text { Non picky eaters } \\
(\mathbf{n = 1 8 0})\end{array}$ \\
\hline Growth, mean (SD) & 12.4 & 14.2 \\
\hline Weight-for-age-percentile: mean SD & 42.8 & 73.61 \\
\hline Less than 15 percentile: $\mathrm{n}(\%)$ & 71 & 22 \\
\hline Weight -for-age-z score & -1.53 & $0.81+-0.70$ \\
\hline Height, mean (SD) & 91.66 & 97.81 \\
\hline Height-for-age-percentile: mean SD & 33.43 & 68.77 \\
\hline Less than 15 percentile: $\mathrm{n}(\%)$ & 36 & 14 \\
\hline Height-for-age-z score & -1.12 & $0.28+-0.41$ \\
\hline BMI, mean +-SD & 14.93 & 16.67 \\
\hline BMI-for-age percentile: mean SD & 33.2 & 62.03 \\
\hline$>15$ percentile: $\mathrm{n}(\%)$ & 38 & 15 \\
\hline BMI-for-age-z score & -0.97 & $0.33+-52$ \\
\hline
\end{tabular}

Table 3. Variations in development quality between picky and non picky eaters

\begin{tabular}{|l|c|c|}
\hline Development variations & $\begin{array}{c}\text { Picky preschoolers } \\
(\mathbf{n = 6 2 0})\end{array}$ & $\begin{array}{c}\text { Non picky preschoolers } \\
(\mathbf{n}=\mathbf{1 8 0})\end{array}$ \\
\hline Learning disability Attention deficit & $130(16.2 \%)$ & $58(7 \%)$ \\
\hline Low learning ability & $95(11.8 \%)$ & $35(4.3 \%)$ \\
\hline Speech development & $62(7.7 \%)$ & $38(4.7 \%)$ \\
\hline Speech delay & $37(4.6 \%)$ & $27(3.3 \%)$ \\
\hline Slow verbal development & $74(9.2 \%)$ & $81(10.1)$ \\
\hline Low social communication & $423(52.8 \%)$ & $217(27 \%)$ \\
\hline Afraid of unfamiliar places & $521(65 \%)$ & $107(13.3 \%)$ \\
\hline Afraid of being alone & $117(14.6 \%)$ & $97(12.1 \%)$ \\
\hline
\end{tabular}

eaters (Table 4). Compared to non- picky group, a significantly lower score of all questionnaire items was found in the picky group (26.6 \pm 3.1, vs. $20.8 \pm 2.8$ ).

\section{Discussion}

The American Academy of Pediatrics (AAP), in addition to many world-wide institutions, stated that ,the importance of early detection of psychomotor delay in preschoolers, can be achieved by repeated systematic screening at ages of 9,18 and 30 months consequently [36]. Therefore, a reliable test to adapt for addressing behavioral and motor development in preschool children is essential [32]. The widely used screening tests is the Denver Developmental Screening Test (DDST) which was first published in 1967 [37]. 
Qazaryan KSY (2019) The clinical link of preschoolers' picky eating behavior with their growth, development, nutritional status, and physical activity in Iraq/ Kurdistan region

Table 4. Presentation levels among physical health status of picky and non picky preschoolers

\begin{tabular}{|l|c|c|}
\hline Variables & $\begin{array}{c}\text { Picky } \\
\text { preschoolers(n=620) }\end{array}$ & $\begin{array}{c}\text { Non picky } \\
\text { preschoolers (n=120) }\end{array}$ \\
\hline Physical activities & & \\
\hline 1-Normal pace walking & $130(16.2)$ & $122(15.2)$ \\
\hline Low level & $580(72.5)$ & $98(12.2)$ \\
\hline Normal & & \\
\hline 2-Sport activities & $133(16.6)$ & $18(2.2)$ \\
\hline Low level & $505(63.1)$ & $102(12.7)$ \\
\hline Normal & & $71(8.8)$ \\
\hline 3-Stair-climbing & $128(16)$ & $493(61.6)$ \\
\hline Low level & $498(62.2)$ & $66(8.2)$ \\
\hline Normal & & $543(67.8)$ \\
\hline 4-Running & $133(16.6)$ & $142(17.7)$ \\
\hline Low level & $577(72.1)$ & $592(74)$ \\
\hline Normal & $295(36.8)$ & \\
\hline General activities (1+2+3+4) & $588(73.5)$ & \\
\hline Poor (less or =2 low level activity) & & \\
\hline Good (More or =1 low level activity) & & \\
\hline
\end{tabular}

Despite of several studies have illustrated the prevalence of picky eaters among preschoolers, a small number have evaluated the correlation of picky eating with pediatric development, physical activity, and health status. In this study we provide an interesting impression of selective preschoolers aged 2-4 years in Iraq/Kurdistan region and consider remarkable thoughts regarding the impact on children's growth, development, physical activity, and disease load. The food preferences, development, and physical activities between picky eaters and non- picky eaters were scored and analyzed statistically. Our study is the first in Iraq to correlate the behaviors of preschoolers with low-quality development and lower performance values of physical activities.

The definition of picky eaters among children is variable. Lots of studies are available regarding to the prevalence of picky eating in childhood with a huge difference was shown [38]. The majority of previous studies use parental views concerning picky children to recognize child's selective eating. The core of definition of picky eating was based on the objective questionnaires of detecting picky eating, was used in our study, by strong existence of picky eating behaviors on four queries. As compared to already published papers, the prevalence of picky behaviors among preschoolers, was higher, at fifty seven percent $[6,7,39]$. A new report indicated three significant parent-reported feeding questions that may identify persistent picky eaters at an early age [40]. Therefore, based on parental views, the three questions included a subjective identification of picky eater by parental thoughts, and two typical and ordinary picky eating behaviors (strong likes concerning food and unwillingness to accept new foods). These two picky eating behaviors were adopted in our questionnaire. We recruited the other two characteristic fussy eating behaviors which could assist to spot remarkable precision of picky eating behaviors among preschoolers.

In Hong Kong, a large longitudinal study revealed that forty three percent among seven thousands children aged 2-7 years, were reported by their parents as being picky eaters, and forty percent of children's picky-eating behavior lasted longer than 2 years [6,20]. A crosssectional survey showed that the percentage of picky eaters increased from nineteen percent at 16 weeks old to fifty percent by age of 2 years [7]. In addition, another cross-sectional survey of Chinese preschoolers addressed that prevalence of picky eating was thirty six percent in
2-3 years olds as compared to twelve percent in 6-11-month-old [8]. Therefore, such results indicate that picky eating is a persistent dilemma [6,7].

In our current study, correlatively more participants in the younger age group were picky eaters $(50 \%$ age $2-3$ years vs. $27.5 \%$ age $3-4$ years). However, pickiness behavior start to decline with age through early childhood and reach its peak time ate age of two-four years old according to previous studies $[4,41]$.

The picky eaters in this group of preschoolers from Kurdistan/Iraq illustrated vital lower numbers and values of accepted foods and food preference respectively. The questionnaire items for the evaluation of pickiness behavior in the current research study was similar to those questionnaires of preceding studies $[7,10,12]$. Therefore, meat, fruit, fish, and specific kinds of vegetables were the food items that they dislike to consume; parallel picky-eating behaviors were observed among children in Hong Kong [39]. the most common picky-eating behaviors among preschoolers in Iraq/Kurdistan region were being unwilling to eat regular meals, refusing fruit and vegetables, and being likely to eat sweets or snacks instead of meals. Such results are identical to picky-eating behaviors conducted in another research study in Singapore [10].

Further research studies revealed that picky eaters were twice as likely to be underweight at 4.5 years old as non-picky eaters [30]. In the current study, excessive milk-drinking, eating sweets and snacks were common picky-eating behaviors. In preschoolers and according to the food records, extreme milk-drinking may result in low appetite at meal-times and cause inadequate energy intake. According to crosssectional analysis in the United Kingdom and based on questionnaires completed by parents when their children were aged 30 months, revealed that seventeen percent eating a partial quality, thirteen percent preferring drinks to food: therefore limited variety and favoring drinks were the most common problem behaviors [3]. Furthermore, the study pointed that an eating problem, in children over 2 years, resulted in underweight over the first 2 years; eleven percent had weight loss compared with three percent of children who were not diagnosed as having an eating trouble. Accordingly, weight loss is more common in picky eaters and excessive milk-drinking may be a cause of low appetite at regular meal-times.

Currently, There are no enough research studies and approved information about the impact of picky-eating behavior on the nutritional and growth status of preschoolers.

A study that compared the weight, height, and weight-for-height percentiles of thirty four children with picky-eating behavior and 136 healthy controls concluded that seven of 34 children (20.6\%) in the picky-eating group and nine of $136(6.6 \%)$ in the control group were underweight; being underweight was found in fifteen children (14.2\%) younger than 3 years old and in one child (1.6\%) older than three years old [42]. The investigators found that children with picky-eating habits are at an increased risk of being underweight, particularly in those younger than 3 years old.

According to our data, weight and height of picky eaters were significantly lower than non-picky eaters: In general, the weight-forage, height-for-age, and BMI-for-age percentiles of non-picky eaters were above 50th, while picky' eaters were under 50th. Also, compared with non-picky eaters, $z$-score of weight-for-age, height-for-age, and BMI-for-age in picky eaters was $0.91,0.73$, and 0.44 SD lower, correspondingly (Table 2 ). 
Furthermore, picky-eaters comprised significantly higher proportions of children who were underweight, short, and with low BMI ( $<15$ percentiles) compared to non-picky eaters.

A negative impact of picky eating behaviors on growth was found in pre-school and early school-age children. However, in a study conducted in Saudi Arabia, 315 pre-school children with eating problems as compared to one hundred health control revealed that the main feeding problems detected were picky eating in $85.5 \%$ of feeding problem with normal growth group, these group children were still having normal growth parameters, but they had significantly lower growth parameters than healthy children [12]. Moreover, in a China study of nine hundred thirty seven recruited healthy children of 3-7 years old concluded the prevalence of picky eating as reported by parents was fifty four percent ; the weight for age $z$-score was significantly lower in picky eaters compared to non-picky eaters [8].

Furthermore, dietary consumption in preschoolers might be negatively influenced by pickiness behavior. A randomized trial of Chinese preschoolers (aged 2.5-5 years) based on validated dietary analysis software of local database of recommended nutrient intakes concluded that median daily energy intake was twenty five percent lower than the age-appropriate intake in preschool picky eaters, and found that preschool picky eaters with low weight-for-height index was at risk for significant dietary and nutrient deficiencies [43]. In that study, almost fifty percent of the picky eaters met the recommendation for daily servings of fruit, and fewer in vegetables (14.7\%) and dietary products (6.3\%), and grains and cereals (6.3\%) [43]. Furthermore, in Chinese study, preschoolers (ages 3-7 years) concluded that picky eaters had lower intakes of protein, dietary fiber, vegetables, fish, and cereals, compared with non-picky eaters (8). Another study of Chinese young infants and toddlers (6 months- 3 years) observed that lower intake of eggs and their food cohort in picky eaters compared to nonpicky eaters [9]. However, in our study, the common dislike foods among preschool picky eaters (ages 2-4 years) were meat, vegetables and fruit $(37 \%, 39 \%, 22 \%)$ respectively.

Besides, a research study of four hundred twenty six German children aged 8-12 years revealed that pickiness behavior was linked to abnormal behavior for instance, anxiety, depression, withdrawal and somatic complaints, as compared to normal behavior in non-picky eaters [4]. The link between numerous eating disorders (overeating, anorexia, or feeding difficulties) and development has been reported in children and adolescents [26,44-48], while the association between picky-eating habits and development in preschool children has rarely been documented.

However, in our study, we found positive correlation between picky-eating behaviors and low- quality general development in preschoolers with unknown reason due to deficient evidence in research and literature. Besides, picky eaters tended to have a greater fear of unfamiliar places as compared to non picky preschoolers. A future longitudinal study and further studies are required to illuminate the underlying consequence relationship between picky eating and low- quality of general development. Also, picky eaters tended to have lower values of the performance in physical activities especially a lowerlevel of stair-climbing. In addition, picky preschoolers tended to have higher risk of constipation and acute infectious illness as compared to non picky preschoolers [49].

Likewise, Picky eaters (aged 3-5 years) with growth faltering who were randomized to receive 3-month oral nutritional supplementation had significantly greater increases in weight and height than non- supplemented controls, and developed proportionally fewer upper respiratory tract infections [50]. In Filipino children, long-standing oral nutritional supplementation helped promote nutritional adequacy and growth who were at risk of nutritional deficiency [51]. The findings showed that long-term use of oral nutritional supplement enhanced food variety and promoted sufficient intake of nutrients that were inadequate in Filipino children's diets without interfering the intake of normal family meals. Therefore, picky preschoolers had improvement in their growth after consuming oral nutritional supplementation [50]. Another interesting randomized controlled trial of Chinese picky preschoolers aged 3-6 years old and weight-for- height $\leq 25$ th percentile showed higher significant changes in growth parameters and nutrient intake in the group with a nutritional milk supplement than the group with nutrition counseling alone [52]. As comparison to the children with nutrition counseling alone, increases in weight- for-age $z$-scores and weight-for-height $z$-scores were significantly better at 3 months, and increases of intakes in energy, protein, carbohydrate, docosahexaenoic acid, arachidonic acid, calcium, phosphorous, iron, zinc, and vitamins (A, C, D, E, B6) were significantly elevated at 2 months and 4 months in the children with a nutritional milk supplement [52].

The power of this study included the population-based design in preschool children and extensive questionnaire to assess picky eating behavior, growth status, quality of development, level of physical activity, and health status. To reduce the selection bias, the participants enrolled were healthy, with good economic state and no need for nutrition support; the caregivers had sufficient knowledge and perception in children's diets, development, and physical activities. Moreover, our study defined the picky eating by objective questionnaires and scored the performance on each questionnaire items of development and physical activity to demonstrate the difference of development and physical activities between picky and non-picky eaters, this to empower the scientific credibility.

Our study has certain limitations. The self-rating questionnaires only presented the point of views from caregivers, over-/underestimation in reporting may exist due to lack of objective behavioral observations on eating behaviors, interaction, developments, and physical activities. lastly, the inclusion of participants from two various places may limit the generalization of the findings to the whole country.

\section{Overall}

Pickiness behaviors in preschoolers might have negative impacts and consequences on development quality, physical activity level, and general health status. Therefore, Parents and caregivers need to be well informed and taught about feeding strategies to enhance adequate food variety for their preschoolers and to increase the number of foods accepted by their toddlers and appropriate dietary interventions to develop sound feeding solutions to address picky-eating behaviors. Also, clinicians might play a vital role to guide and support parents and caregivers on the finest approaches to reach the best possible nutrition for their children who are picky eaters, and early diagnosis and clinical intervention of pickiness behavior among preschoolers might help to reduce or limit the negative impacts of such behavior on children's growth and development.

\section{Availability of data and materials}

Data for research purposes are available upon request.

\section{Consent for publication}

All participants gave and provided their written consent forms. 
Qazaryan KSY (2019) The clinical link of preschoolers' picky eating behavior with their growth, development, nutritional status, and physical activity in Iraq/ Kurdistan region

\section{Conflict of interest statement}

The authors declare that the research was conducted in the absence of any commercial or financial relationships that could be construed as a potential conflict of interest.

\section{Acknowledgement}

We thank and acknowledge all children and their parents or caregivers for their time and efforts to take part in this research project.

\section{Funding}

This study was not funded by grants or other financial sponsors. The authors declare that they have no any financial arrangement with a company whose product is discussed in the manuscript.

\section{References}

1. Jacobi C, Schmitz G, Agras WS (2008) Is picky eating an eating disorder?. Int J Eat Disord 41: 626-634.

2. Galloway AT, Fiorito L, Lee Y, Birch LL (2005) Parental pressure, dietary patterns, and weight status among girls who are "picky eaters". J Am Diet Assoc 105: 541-548.

3. Wright CM, Parkinson KN, Shipton D, Drewett RF (2007) How do toddler eating problems relate to their eating behavior, food preferences, and growth? Pediatrics 120 : e1069-1075.

4. Jacobi C, Agras WS, Bryson S, Hammer LD (2003) Behavioral validation, precursors, and concomitants of picky eating in childhood. J Am Acad Child Adolesc Psychiatry 42: $76-84$.

5. Nicholls D, Bryant-Waugh R (2009) Eating disorders of infancy and childhood: definition, symptomatology, epidemiology, and comorbidity. Child Adolesc Psychiatr Clin N Am 18: 17-30

6. Mascola AJ, Bryson SW, Agras WS (2010) Picky eating during childhood: a longitudinal study to age 11 years. Eating behaviors 11: 253-257.

7. Carruth BR, Ziegler PJ, Gordon A, Barr SI (2004) Prevalence of picky eaters among infants and toddlers and their caregivers' decisions about offering a new food. $J \mathrm{Am}$ Diet Assoc 104: 57-64.

8. Xue Y, Zhao A, Cai L, Yang B, Szeto IM, et al. (2015) Growth and development in Chinese pre-schoolers with picky eating behaviour: a cross-sectional study. Plos one 10: e0123664.

9. Li Z, van der Horst K, Edelson-Fries LR, Yu K, You L, et al. (2017) Perceptions of food intake and weight status among parents of picky eating infants and toddlers in China: a cross-sectional study. Appetite 108: 456-463.

10. Goh DY, Jacob A (2012) Perception of picky eating among children in Singapore and its impact on caregivers: a questionnaire survey. Asia Pacific family medicine 11: 5.

11. Micali N, Simonoff E, Elberling H, Rask CU, Olsen EM, et al. (2011) Eating patterns in a population-based sample of children aged 5 to 7 years: association with psychopathology and parentally perceived impairment. J Dev Behav Pediatr 32: 572580 .

12. Shim JE, Kim J, Mathai RA (2011) STRONG Kids Research Team. Associations of infant feeding practices and picky eating behaviors of preschool children. J Am Diet Assoc 111: 1363-1368.

13. Chatoor I, Ammaniti M (2007) Classifying feeding disorders of infancy and early childhood. Age and gender considerations in psychiatric diagnosis: A research agenda for DSM-V 2007: 227-242.

14. Kerzner B (2009) Clinical investigation of feeding difficulties in young children: a practical approach. Clinical pediatrics 48: 960-965.

15. Hegazi MA, Sehlo MG, Al-Jasir A, El-Deek BS (2015) Development and cognitive functions in S audi pre-school children with feeding problems without underlying medical disorders. J Paediatr Child Health 51: 906-912.

16. Chen W, Chang MH (2010) New growth charts for Taiwanese children and adolescents based on World Health Organization standards and health-related physical fitness. Pediatrics \& Neonatology 51: 69-79.

17. Goncalves EA, Moreira EB (2013) Trindade, G.M. Fiates Eating disorders in childhood and adolescence. Rev Paul Pediatr 31: 96-103.
18. Steyn NP, Nel JH, Nantel G, Kennedy G, Labadarios D (2006) Food variety and dietary diversity scores in children: are they good indicators of dietary adequacy?. J Public Health 9: 644-650.

19. Woolston JL (1983) WoolstonEating disorders in infancy and early childhood. $\mathrm{J} \mathrm{Am}$ Acad Child Psychiatry 22: 114-121.

20. Kotler LA, Cohen P, Davies M, Pine DS, Walsh BT (2001) Longitudinal relationships between childhood, adolescent, and adult eating disorders. J Am Acad Child Adolesc Psychiatry 40: 1434-1440.

21. McDermott BM, Mamun AA, Najman JM, Williams GM, O'Callaghan MJ, et al (2010) Longitudinal correlates of the persistence of irregular eating from age 5 to 14 years. Acta Paediatr 99: 68-71.

22. Nicklaus S, Boggio V, Chabanet C, Issanchou S (2010) A prospective study of food variety seeking in childhood, adolescence and early adult life. Appetite 2005 Jun 1;44(3):289-97.

23. Dubois L, Farmer A, Girard M, Peterson K, Tatone-Tokuda F (2007) Problem eating behaviors related to social factors and body weight in preschool children: A longitudinal study. Int J Behav Nutr Phys Act 4: 9.

24. Benton D. The influence of dietary status on the cognitive performance of children. Mol Nutr Food Res 54: 457-470.

25. McAfee AJ, Mulhern MS, McSorley EM, Wallace JM, Bonham MP, et al. (2012) Intakes and adequacy of potentially important nutrients for cognitive development among 5-year-old children in the Seychelles Child Development and Nutrition Study. Public health nutrition 15: 1670-1677.

26. Chatoor I, Surles J, Ganiban J, Beker L, Paez LM, et al. (2004) Failure to thrive and cognitive development in toddlers with infantile anorexia. Pediatrics 113: e440-e447.

27. Department of Health, Committee on Medical Aspects of Health (1989) The diets of British school children. Sub-committee on nutritional surveillance. Committee on medical aspects of food policy. Rep Health Soc Subj (Lond) 36: 1-293.

28. McPherson RS, Hoelscher DM, Alexander M, Scanlon KS, Serdula MK (2000) Dietary assessment methods among school-aged children: validity and reliability. Preventive medicine 31: S11-33.

29. Wardle J, Guthrie CA, Sanderson S, Rapoport L (2001) Development of the children's eating behaviour questionnaire. J Child Psychol Psychiatry 42: 963-970.

30. Dubois L, Farmer AP, Girard M, Peterson K (2007) Preschool children's eating behaviours are related to dietary adequacy and body weight. Eur J Clin Nutr 61: 846855 .

31. Frankenburg WK, Camp BW, Van Natta PA, Demersseman JA, Voorhees SF (1971) Reliability and stability of the Denver developmental screening test. Child Development 1: $1315-1325$.

32. Wijedasa D (2012) Developmental screening in context: adaptation and standardization of the Denver Developmental Screening Test-II (DDST-II) for Sri Lankan children. Child: care, health and development 38: 889-899.

33. De-Andrés-Beltrán B, Rodríguez-Fernández ÁL, Güeita-Rodríguez J, Lambeck J (2015) Evaluation of the psychometric properties of the Spanish version of the Denver Developmental Screening Test II. Eur J Pediatr 174: 325-329.

34. Hallioglu O, Topaloglu AK, Zenciroglu A, Duzovali O, Yilgor E, et al. (2001) Denver developmental screening test II for early identification of the infants who will develop major neurological deficit as a sequalea of hypoxic-ischemic encephalopathy. Pediatr Int 43: 400-404.

35. Reilly JJ, Penpraze V, Hislop J, Davies G, Grant S, et al. (2008) Objective measurement of physical activity and sedentary behaviour: review with new data. Arch Dis Child 93: 614-619.

36. Council on Children with Disabilities. Section on Developmental Behavioral Pediatrics, Bright Futures Steering Committee, Medical Home Initiatives for Children with Special Needs Project Advisory Committee (2006) Identifying infants and young children with developmental disorders in the medical home: an algorithm for developmental surveillance and screening. Pediatrics 118: 405-420.

37. Sand N, Silverstein M, Glascoe FP, Gupta VB, Tonniges TP, et al. (2005) Pediatricians' reported practices regarding developmental screening: do guidelines work? Do they help?. Pediatrics 116: 174-179.

38. Brown CL, Vander Schaaf EB, Cohen GM, Irby MB, Skelton JA (2016) Association of picky eating and food neophobia with weight: a systematic review. Childhood Obesity 12: $247-262$. 
Qazaryan KSY (2019) The clinical link of preschoolers' picky eating behavior with their growth, development, nutritional status, and physical activity in Iraq/ Kurdistan region

39. Centre for Health Education and Health Promotion, Faculty of Medicine, Chinese University of Hong Kong. Joint Hands to Create a Healthy Eating Culture in School Children (2006)

40. Toyama H, Agras WS (2016) A test to identify persistent picky eaters. Eating behaviors 23: 66-69.

41. Rozin P (1990) Development in the food domain. Dev Psychol 26: 555-562.

42. Ekstein S, Laniado D, Glick B (2010) Does picky eating affect weight-for-length measurements in young children? Clinical pediatrics 49: 217-220.

43. Volger S, Sheng X, Tong LM, Zhao D, Fan T, et al. (2017) Nutrient intake and dietary patterns in children 2.5-5 years of age with picky eating behaviours and low weight-forheight. Asia Pac J Clin Nutr 26: 104-109.

44. French SA, Perry CL, Leon GR, Fulkerson JA (1995) Changes in psychological variables and health behaviors by dieting status over a three-year period in a cohort of adolescent females. J Adolesc Health 16: 438-447.

45. Monge-Rojas R, Nuñez HP, Garita C, Chen-Mok M (2002) Psychosocial aspects of Costa Rican adolescents' eating and physical activity patterns. J Adolesc Health 31: 212-219.

46. Palladino RR, Cunha MC, Souza LA (2007) Language and eating problems in children: co-occurrences or coincidences?. Pro Fono 19: 205-214.
47. Epstein LH, Roemmich JN, Stein RI, Paluch RA, Kilanowski CK (2005) The challenge of identifying behavioral alternatives to food: clinic and field studies. Ann Behav Med 30: 201-209.

48. Bergh C, Brodin U, Lindberg G, Södersten P (2002) Randomized controlled trial of a treatment for anorexia and bulimia nervosa. Proceedings of the National Academy of Sciences 99: 9486-9891.

49. Al-Hazzaa HM, Abahussain NA, Al-Sobayel HI, Qahwaji DM, Musaiger AO (2011) Physical activity, sedentary behaviors and dietary habits among Saudi adolescents relative to age, gender and region. Int J Behav Nutr Phys Act 8: 140.

50. Alarcon PA, Lin LH, Noche Jr M, Hernandez VC, Cimafranca L, et al. (2003) Effect of oral supplementation on catch-up growth in picky eaters. Clinical pediatrics 42 : 209-217.

51. Huynh DT, Estorninos E, Capeding MR, Oliver JS, Low YL, et al. (2016) Impact of long-term use of oral nutritional supplement on nutritional adequacy, dietary diversity, food intake and growth of Filipino preschool children. J Nutr Sci 5.

52. Sheng X, Tong M, Zhao D, Leung TF, Zhang F, et al. (2014) Randomized controlled trial to compare growth parameters and nutrient adequacy in children with picky eating behaviors who received nutritional counseling with or without an oral nutritional supplement. Nutr Metab Insights 7: NMI-S15097.

Copyright: $@ 2019$ Qazaryan KSY. This is an open-access article distributed under the terms of the Creative Commons Attribution License, which permits unrestricted use, distribution, and reproduction in any medium, provided the original author and source are credited. 